\title{
HEPTAGONAL SYSTEMS OF EIGHT LINES IN A PLANE*
}

\author{
BY LOUISE D. CUMMINGS
}

1. Introduction. This paper concerns the determination of the non-equivalent systems of eight real lines in a plane and the investigation is limited to that subdivision of the problem where in a system of eight lines, some seven form a convex heptagon. The eight marks of a system are used to show the non-equivalence of the fifteen heptagonal systems derived in this paper. The notation and method employed are explained fully in the two papers $\dagger$ cited below.

2. The Cases Considered. Two subdivisions of the problem are considered:

CASE I. The secant crosses the heptagon.

CASE II. The secant lies outside the heptagon.

CAsE I. The secant crosses two primary, two secondary, two tertiary, and one quaternary segment. The two primary segments may be (i) adjacent, (ii) alternate, (iii) opposite sides of the heptagon.

Case I furnishes ten non-equivalent systems, (1), (2), (3), (4), (5), (6), (7), (8), (9), (10), tabulated below in Table I.

CAsE II. The secant crosses the segments as follows: (i) seven quaternary, (ii) five quaternary and two tertiary, (iii) three nonconsecutive quaternary and four tertiary, (iv) three consecutive quaternary and four tertiary, (v) one quaternary and six tertiary, and furnishes five non-equivalent systems (11), (12), (13), (14), (15), respectively tabulated in Table I. If the secant crosses (vi) two secondary, four tertiary and one quaternary; (vii) two secondary, two tertiary and three quaternary; (viii) four secondary, two tertiary and one quaternary; three systems are obtained equivalent respectively to systems (3), (4), (2). These results were to be expected since now a second heptagon including the secant line $l_{8}$ exists in the system and this may be used as basic heptagon (see Fig. 1).

* Presented to the Society, March 26, 1932.

$\dagger \mathrm{H}$. S. White, The plane figure of seven real lines, this Bulletin, vol. 38 (1932), pp. 59-65; Louise D. Cummings, Hexagonal systems of seven lines in a plane, this Bulletin, vol. 38 (1932), pp. 105-110. 


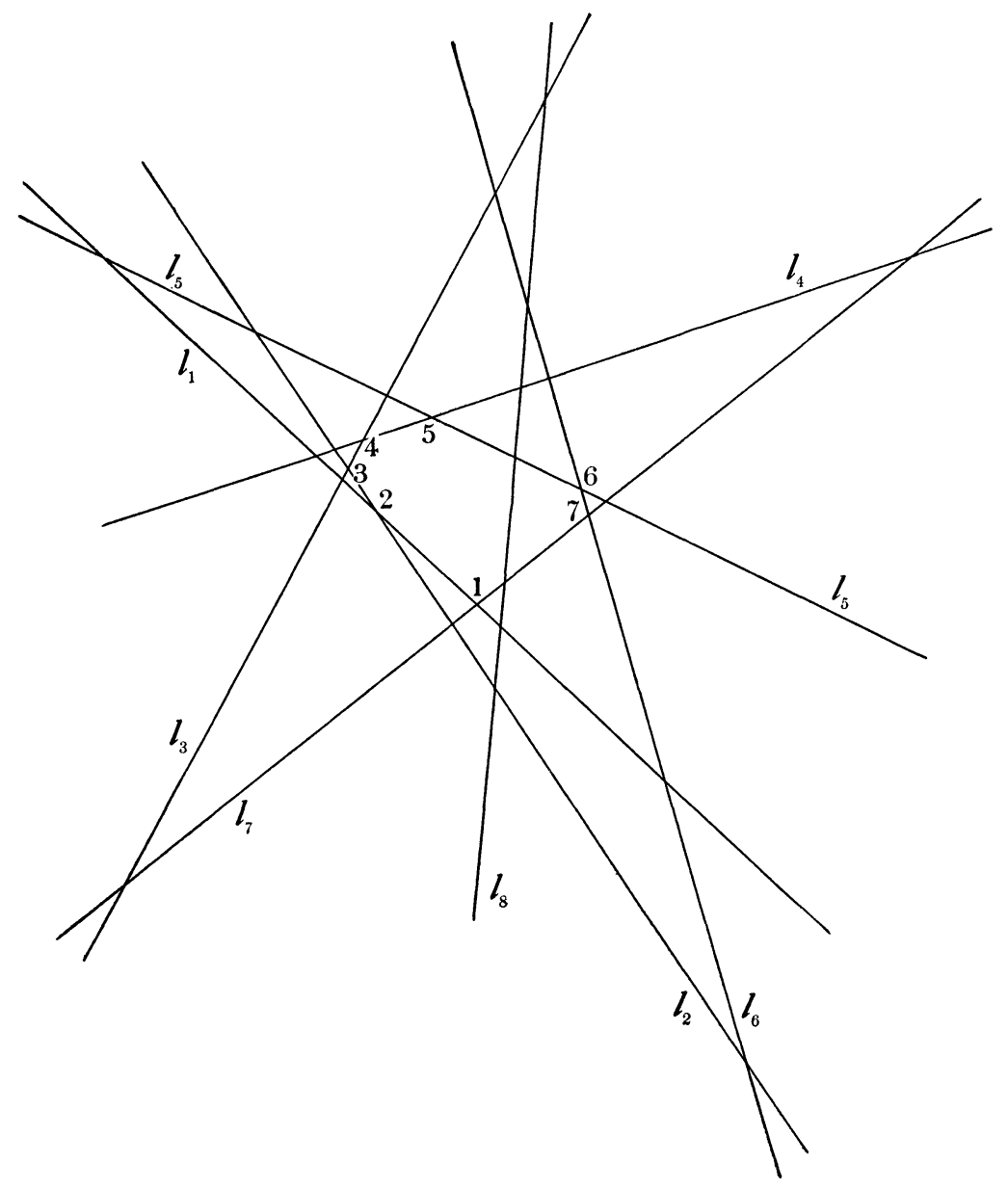

Figure 1. System (3) 
TABLE I

\begin{tabular}{|c|c|c|c|c|c|c|c|}
\hline System & Characteristics & \multicolumn{6}{|c|}{ Polygons } \\
\hline (1) & $(4111)^{8}$ & 1 & 0 & 0 & 0 & 20 & 8 \\
\hline (11) & $(70)+(43)^{7}$ & 0 & 1 & 0 & 7 & 7 & 14 \\
\hline (15) & $(61)^{5}+(421)^{2}+(4111)$ & 0 & 1 & 0 & 5 & 11 & 12 \\
\hline (12) & $(52)+(43)^{3}+(421)^{4}$ & 0 & 1 & 0 & 5 & 11 & 12 \\
\hline (14) & $(61)^{2}+(43)^{2}+(421)^{4}$ & 0 & 1 & 0 & 5 & 11 & 12 \\
\hline (13) & $(421)^{6}+(4111)+(2221)$ & 0 & 1 & 0 & 3 & 15 & 10 \\
\hline (3) & $(511)+(421)^{2}+(4111)^{2}+(3211)^{3}$ & 0 & 1 & 0 & 2 & 17 & 9 \\
\hline (4) & $(421)^{2}+(4111)^{2}+(322)+(3211)^{3}$ & 0 & 1 & 0 & 2 & 17 & 9 \\
\hline (2) & $(4111)^{4}+(3211)^{4}$ & 0 & 1 & 0 & 1 & 19 & 8 \\
\hline (6) & $(70)+(511)^{2}+(43)+(331)^{2}+(3211)^{2}$ & 0 & 0 & 1 & 5 & 12 & 11 \\
\hline (7) & $(52)+(511)+(421)+(4111)+(331)^{2}$ & & & & & & \\
\hline & $+(3211)^{2}$ & 0 & 0 & 1 & 4 & 14 & 10 \\
\hline (5) & $(511)^{2}+(43)+(421)+(331)+(3211)^{3}$ & 0 & 0 & 1 & 4 & 14 & 10 \\
\hline (9) & $(511)+(421)+(4111)+(322)+(3211)^{4}$ & 0 & 0 & 1 & 3 & 16 & 9 \\
\hline (10) & $(511)^{2}+(4111)+(331)+(3211)^{4}$ & 0 & 0 & 1 & 3 & 16 & 9 \\
\hline (8) & $(4111)^{3}+(3211)^{4}+(2221)$ & 0 & 0 & 1 & 2 & 18 & 8 \\
\hline
\end{tabular}

3. The Figure. Examination of the basic diagram shows that a secant which intersects four secondary segments must also intersect two tertiary segments. Therefore all types of paths for the eighth line have been investigated in the preceding eighteen cases.

The numbers listed for the polygonal divisions of the plane for these fifteen heptagonal systems show pairs of systems, for example (3) and (4), with the same polygonal numbers but with different characteristics. Therefore equality of polygonal numbers is a necessary but not a sufficient condition for the equivalence of two systems.

4. Conclusion. This method of analysis of straight-line nets by the contiguous segments as herein extended to all the lines in the system regardless of their relation to a heptagon or other basal polygon is applicable to any number $n$ of straight lines and is even not restricted to the case that only two lines shall pass through a point. This method simplifies the discovery of the substitution which transforms one of two equivalent systems into the other.

Vassar College 